\title{
Querying the public databases for sequences using complex keywords contained in the feature lines Olivier Croce*†, Michaël Lamarre ${ }^{\dagger}$ and Richard Christen
}

Address: Laboratoire de Biologie Virtuelle, UMR 6543, CNRS \& University of Nice Sophia-Antipolis, Centre de Biochimie, Parc Valrose, Nice, F06108, France

Email: Olivier Croce* - croce@unice.fr; Michaël Lamarre - lamarre@unice.fr; Richard Christen - christen@unice.fr

* Corresponding author †Equal contributors

Published: 27 January 2006

BMC Bioinformatics 2006, 7:45 doi:10.1|86/|47|-2/05-7-45

This article is available from: http://www.biomedcentral.com/I47I-2105/7/45

(C) 2006 Croce et al; licensee BioMed Central Ltd.

This is an Open Access article distributed under the terms of the Creative Commons Attribution License (http://creativecommons.org/licenses/by/2.0), which permits unrestricted use, distribution, and reproduction in any medium, provided the original work is properly cited.
Received: 03 October 2005

Accepted: 27 January 2006

\begin{abstract}
Background: High throughput technologies often require the retrieval of large data sets of sequences. Retrieval of EMBL or GenBank entries using keywords is easy using tools such as ACNUC, Entrez or SRS, but has some limitations, in particular when querying with complex keywords.

Results: We show that Entrez has severe limitations with respect to retrieving subsequences. SRS works well with simple keywords but not with keywords composed of several terms, and has problems with complex queries. ACNUC works well, but does not allow precise queries in the Feature qualifiers. We developed specific Perl scripts to precisely retrieve subsequences as defined by complex descriptors in the Features qualifiers of the EMBL entries. We improved parts of the bioPerl library to allow parsing of large data files, and we embedded these scripts in a user friendly interface (OS independent) for easy use.

Conclusion: Although not as fast as the public tools that use prebuilt indexes, parsing the complete entries using a script is often necessary in order to retrieve the exact data searched for. Embedding in a user friendly interface allows biologists to use the scripts, which can easily be modified, if necessary, by bioinformaticians for unforeseen needs.
\end{abstract}

\section{Background}

The quantity of biological information available in the public databases is now very large and doubling nearly every year [1]. Projects involving high throughput data from approaches such as the new transcriptomic or genomic technologies require large quantities of data to be dealt with. For example, in order to design a DNA chip for bacterial identification, one may want to retrieve every available sequence for a universal gene such as the $16 \mathrm{~S}$ rRNA gene (nearly 200,000 sequences expected by the end of year 2005).
Retrieval of such sequences is not trivial. Retrieval by sequence similarity is not feasible, since some of these genes are very variable (ITS regions for example). Also it is difficult, if not impossible, to determine a cutoff level in order to exclude non homologous gene sequences. Blast [2] for example allows a cutoff according to the "Evalue", while Exonerate [3] also allows a cutoff on percentage of similarity. The "E value" cutoff depends on the database size that changes every day. The percentage of similarity is better, but for divergent ubiquitous genes, experiments show that some homologous sequences are not retrieved while non homologous sequences are retrieved. Impor- 
Table I: Keyword variations and mis-spelling.

\begin{tabular}{|c|c|}
\hline ITSI, INTERNAL TRANSCIBED SPACER I & I seqs. \\
\hline ITSI, INTERNAL TRANSCRIBED SPACER I & 22 seqs. \\
\hline INTERNAL TRANSCRIBED SPACER I, ITSI & 32 seqs. \\
\hline INTERNAL TRANSCRIBED SPACER I (ITSI) & 166 seqs. \\
\hline INTERNAL TRANSCRIBED SPACER I & 70,663 seqs. \\
\hline INTERNAL TRANSCRIBED SPACER I & 15 seqs. \\
\hline INTERNAL TRANSCRIBED SPACER I OF RIBOSOM & 9 seqs. \\
\hline INTERNAL TRANSCRIBED SPACER I; ITS I & 4 seqs. \\
\hline INTERNAL TRANSCRIBED SPACER I (ITSI) OF & 2 seqs. \\
\hline INTERNAL TRANSCRIBED SPACER ITSI OF RIBO & I seqs. \\
\hline INTERNAL TRANSCRIBED SPACER I (ITSI) OR & I seqs. \\
\hline INTERNAL TRANSCRIBED SPACER I & 2 seqs. \\
\hline INTERNAL TRANSCRIBED SPACER I; ITSI & 65 seqs. \\
\hline INTERNAL TRANSCRIBED SPACER I; ITS-I & 65 seqs. \\
\hline INTERNAL TRANSCRIBED SPACER ITSI & 22 seqs. \\
\hline INTERNAL TRANSCRIBED SPACER I ITSI & 45 seqs. \\
\hline INTERNAL TRANSCRIBED SPACER I; 5.8S RIBO & 29 seqs. \\
\hline INTERNAL TRANSCRIBED SPACER I, 5.8S RIBO & 65 seqs. \\
\hline INTERNAL TRANSCRIBED SPACER REGION I & 35 seqs. \\
\hline INTERNAL TRANSCRIBED SPACER REGION 2 & 35 seqs. \\
\hline INTERNAL TRANSRIBED SPACER I & I seqs. \\
\hline INTERNAL TRANSCIBED SPACER I & 6 seqs. \\
\hline CONTAINS INTERNAL TRANSCRIBED SPACER I, & 368 seqs. \\
\hline INTERNAL TRANSRCIBED SPACER I & 10 seqs. \\
\hline RIBOSOMAL INTERNAL TRANSCRIBED SPACER I & 42 seqs. \\
\hline INTERNAL TRANCSRIBED SPACER I & I seqs. \\
\hline CONTAINS INTERNAL TRANSCRIBED SPACER I A & I seqs. \\
\hline INTERNAL TRANSCRIBED SPACER I AND 5.8S R & 45 seqs. \\
\hline INTERNAL TRNSCRIBED SPACER I & I seqs. \\
\hline INTERNAL TRANSCRIBER SPACER I & I seqs. \\
\hline INTERNAL TRANSCRIBED SPACER I TYPE I & I seqs. \\
\hline INTERNAL TRANSCRIBED SPACER I TYPE II & I seqs. \\
\hline INTERNAL TRANCRIBED SPACER I & I seqs. \\
\hline INTERNAL TRANSCRIBED SPACER I (ITSI) REG & 12 seqs. \\
\hline ITSI INTERNAL TRANSCRIBED 2 & I seqs. \\
\hline INTERNAL TRANSCRIBED SPACER (ITSI) & 16 seqs. \\
\hline INTERNAL TRANSCRIBED SPAVER I & 119 seqs. \\
\hline
\end{tabular}

ACNUC was used to retrieve in the entire EMBL release 84 (all organisms), existing variations in features annoted with "internal transcribed spacer I" (responses are truncated on the right). We can observe large variations as well as mis-spelling. For confirmation purpose, we queried EMBL release 84 for keyword "ITSI". ACNUC returned I6,392 entries while SRS at EBI returned I6, I35 entries, due to problems with spaces and extra characters such as commas.

tantly, a similarity search may return only parts of sequences, and it is difficult to automatically retrieve entire sequences.

The best solution seems to be a retrieval based on keywords that describe the particular feature to retrieve. This is however not always easy. Keywords used to describe a particular feature may be diverse and sometimes misspelled. The entire list of keywords can be retrieved (with ACNUC for example), parsed and painfully analysed to build a complete list of keywords. This task is more difficult with queries composed of complex keywords containing several words or numbers. Popular tools such as ACNUC [4], Entrez [5] or SRS [6] have been designed for the purpose of querying with keywords, but we show in this paper that they should be used with care and caution and that they still have flaws for precisely retrieving sequences according to a complex keyword. This is known by bioinformaticians working in this field who often use their own parsers to directly analyse entries, and in part led to the development of the bio-(Java, Perl, Python) libraries to name only the most commonly used. However, these wide purpose libraries have problems in particular for parsing large data files. We have developed a derivative of the bioPerl library and embedded it in a user friendly interface that makes it possible to easily define complex keywords, the fields to search for and to define the order for searching in these fields. Subsequences are automatically extracted and stored as files or directly in a "biosql" standard relational database for easy management.

\section{Implementation (EmblEx)}

EmblEx is composed of three parts: i) a series of perl scripts, ii) a MySQL database and iii) a user-friendly graphic interface for queries through a web browser.

Emblex searches in ASCII files downloaded from a ftp server such as that of EBI [7]. Their URL can be stored by EmblEx, and files can be automatically downloaded and uncompressed with EmblEx, an internal routine allows to check if this file had been updated after the previous download.

Users can then define complex keywords (or regular expressions) to be searched for, the feature keys to search in, in which order, and the output format. Subsequences and related information are then extracted for the specific feature starting from the first feature as indicated in EmblEx. The available BioPerl module for extracting data from EMBL files was found to be too slow and replaced by a dedicated extractor. Complex feature entries, such as those containing the join() and complement() operators, or those using the "acc_no:xxx..xxx" syntax, where one feature calls upon a fragment of another sequence, are properly processed by EmblEx. Possible outputs are ASCII, HTML and within a MySQL database in BioSQL specification. Indeed, BioSQL is an emerging standard for biological data [8]. Its substantial advantage is the large number of scripts already developed within the BioPerl, BioPython, BioJava communities. The bioperl-db script used to load the database, originally developed for Linux systems only [9] was ported to MS Windows.

Because it may be difficult for many biologists to use scripts from the command line, we imbedded our scripts within a user friendly interface. EmblEx's interface was developed in HTML in order to be compatible with any systems and web browser. 
Files available for download from our server [10] include every necessary dependency and are easily installed on every system. An online version is also available on our web server (no installation is required but some features have been disabled such as BioSQL database and update module).

\section{Results}

We became aware of a problem when we were not able to retrieve some sequences with SRS, yet we knew they were in the EMBL database. For the purpose of the demonstration, we will in this paper focus on the retrieval of nuclear ribosomal intervening sequences (ITS, a short domain located between the small and large ribosomal RNA subunits, transcribed) which are widely used for the purpose of bacterial and protozoan identification [11-23]. We will restrict our experiment to the keyword "internal transcribed spacer 1", although we know that this region is also described using alternate keywords (Table 1)

For simplicity (the whole public database changes every day), comparative queries were done on EMBL release 84 (September 2005). We wanted to search only within features: "misc_feature", "misc_rna", "gene", "rrna", "intron" and "source", to exclude retrieval of amplification primers for example. If the internal transcribed spacer 1 is described both in the "source" and "misc_rna" Feature keys, we wanted to extract the subsequence as defined by the boundaries indicated in the "misc_rna" qualifier. Finally, for simplicity, when an entry contained more than one ITS domain, only the parent entry accession number was considered (link to parent database with SRS, scripting with python for the other tools).

\section{I/Entrez}

Among biologists, Entrez is probably the most popular tool to retrieve sequences (and other data). It is poorly adapted for our purpose as it inefficiently scans the feature lines and cannot extract subsequences. Without subsequence extraction according to the boundaries described in the features lines, we would have to use a dedicated parser anyway.

We tried "Fungi" [Organism] AND srcdb_genbank [PROP] AND "internal transcribed spacer 1" [Feature key] AND gene_in_genomic [PROP].

This returned 28,592 entries (note that it may change everyday as Entrez does not allow a query on the release or update databases).

\section{2/SRS}

SRS is a particularly powerful tool. Using the extended web form, it is possible to easily build powerful queries. The internal getz langage can also be used either from the command line or using the "Result" page. Using the getz alternative, it is possible to use regular expressions. Several queries were tried (in its "Result" section, SRS reformulates these queries differently, but they seem to run faster as written below).

[emblrelease-Division:fun] $>$ ([emblreleaseFtKey:gene|intron|misc_feature|misc_rna|rrna|source] \& [embl-FtDescription:internal transcribed spacer 1])

This query returned an "Error: No entries found " message (but see below). Replacing "internal transcribed spacer 1" by "internal?transcribed?spacer?1" returned the same message. We then used the wild card "*" instead of "?" and obtained the same message.

We had to use the AND ("\&") operator to obtain a response.

Q1: [emblrelease-Division:fun] > ([emblreleaseFtKey:gene|intron|misc_feature|misc_rna|rrna|source] \& [emblrelease-FtDescription:internal\&tran-

scribed\&spacer\&1])

This query took about two minutes and returned 33,767 entries and was reformulated by SRS as shown below:

Query "([emblrelease-Division:fun] > $(((()[$ emblreleaseFtKey:gene] | [emblrelease-FtKey:intron]) | [emblreleaseFtKey:misc_feature]) | [emblrelease-FtKey:misc_rna]) | [emblrelease-FtKey:rrna]) | [emblrelease-FtKey:source]) \& ((([emblrelease-FtDescription:internal] \& [emblreleaseFtDescription:transcribed]) \& [emblrelease-FtDescription:spacer]) \& [emblrelease-FtDescription:1]))).

Since we suspected that more entries could be obtained we also tried:

Q2: [emblrelease-Division:fun] > ([emblreleaseFtKey:gene|intron|misc_feature|misc_rna|rrna|source] \& [emblrelease-FtDescription:internal\&tran-

scribed\&spacer\& $\left.1^{*}\right]$ )

After a very long time, this query returned an error message in the form of an error of memory allocation "Error: Insufficient memory - error during malloc ". The query was tried on week end, when the EBI server is less busy but it returned the same message; other servers offering SRS [24] also returned the same error message. When asked, EBI people answered that too many data had to be treated for the memory size of their server.

We then tried to use a regular expression, which took a very long time and returned an error message: 
Table 2: Compararison of results obtained using similar queries and different tools or servers.

\begin{tabular}{lrrrrr}
\hline & total & SRS ebi & EmblEx & SRS infobiogen & ACNUC \\
\hline SRS ebi & 33,758 & & 64 & 24,520 & 71 \\
EmblEx & 33,697 & 2 & & 24,495 & 15,145 \\
SRS infobiogen & 24,346 & 15,108 & 15,145 & 24,491 & \\
ACNUC & 33,692 & 5 & 3 &
\end{tabular}

The first column indicates how many different accession numbers were returned in each case. Other columns indicate how many of these accession numbers were not retrieved by the other procedures.

Q3: [emblrelease-Division:fun] > ([emblreleaseFtKey:gene|intron|misc_feature|misc_rna|rrna|source] \& [emblrelease-FtDescription:/internal transcribed spacer 1/ ])

The following query also returned an error message.

[emblrelease-Division:fun] $>$ ([emblreleaseFtKey:gene|intron|misc_feature|misc_rna|rrna|source] \& [emblrelease-FtDescription:/internal transcribed spacer/]

Finally a query with /spacer/found 62,863 entries, a query with "/internal/" found 60,637 entries and queries with "/ internal spacer/" or "/internal|sspacer/" both returned a message "Error: No entries found " demonstrating that (on ech public server tried) SRS has a problem with spaces, both in the extended form and regular expressions (see the discussion).

The same queries were done on the Infobiogen server, the same day (September 16th, 2005). These queries returned very different numbers of entries (Table 2). Parse and analysis of the results (see below) showed a potential problem with SRS at Infobiogen. When asked, the Infobiogen people answered, acknowledging a problem with the SRS indexes. SRS did not return an error message, but the entries obtained were globally wrong; among the 15,108 response unique to SRS at Infobiogen (see below) we found for example accession number AB011433, which refers to a protein coding sequence.

\section{IACNUC}

For querying with ACNUC we used the " raa_query" client (available on the pbil server [25]). When we started this analysis, we found that ACNUC had a bug that did not allow it to find complex keywords spanning two lines. When informed, ACNUC people corrected this bug, and the present queries were done on ACNUC after correction (September 16th, 2005).

A first list was built with command se-sp = fungi, a second list was built with command se-o = nuclear; intersection of the two lists returned 866,080 results. The -o option is necessary as ACNUC stores as "fungi" the mitochondrial and nucleomorph sequences (no chloroplastic sequence known in fungi). Intersection with " $k=$ division fun" was then necessary in order to exclude sequences from the "ENV" division. Finally, it would have been possible to use intersections with successive queries in the form " $\mathrm{k}=$ misc_rna" in order to include only the proper FT keys, and then to proceed to an union of the lists, but this can be tedious and we have no direct possibility to choose among the fields "source" and "misc_rna" to extract the proper subsequence according to the boundaries described in the most specific feature.

A recent implementation in ACNUC allows to scan specific lines such as the FT lines. This is much slower, as ACNUC cannot use its index tables, but allows a more precise selection. The final list was queried using command mo -7 and searching FT lines for "internal transcribed spacer $1 "$. This query took about three minutes and returned 33, 692 entries. ACNUC did not find seven entries found by EmblEx (X93976, X93980, X93987, Z48813, Z48817, Z48818, Z48819), due to a problem discussed below.

\section{4/Extraction using a dedicated script}

We used EmblEx to parse the EMBL fun.dat file, which contains all of the fungi sequences (release 84), for the presence of "internal transcribed spacer $1 "$ in the following features: misc_feature, misc_rna, gene, rrna, intron or source, in that order. This query took seven minutes and returned 33,696 entries.

\section{5/Analysis}

A python script allowed to parse the results and to analyse which accession numbers were retrieved by one of the tools and not retrieved by another one (Table 2). As shown in this table, ACNUC, SRS on EBI and our Perl script had similar, but different results. As indicated above, SRS on Infobiogen returned wrong results because of index problems. Next we looked for entries retrieved only by a single tool (Table 3). SRS at EBI had 27 such entries, EmblEx none and ACNUC 3.

The 64 SRS entries not found by EmblEx (including the 27 entries unique to SRS EBI) were manually scanned and results indicated in table 4 . All of the entries contain the keywords searched for, but either with other words in 
Table 3: Unique answers.

\begin{tabular}{lr}
\hline SRS ebi & 27 \\
EmblEx & 0 \\
SRS infobiogen & 15,108 \\
ACNUC & 3
\end{tabular}

This table shows how many accession numbers are unique to a query (retrieved only by a single tool).

between or in the wrong order. The entries specific to ACNUC were also examined. The complex keyword was easily found, but in wrong features ("snorna" or "precursor_rna" for example).

The seven entries found by EmblEx and not by ACNUC corresponded to entries having "internal transcribed spacer 1" in the proper feature, but that had been submitted as "unknown" organism, latter corrected to "fungi" but without any change of the DT "last updated" field. Since ACNUC updates its databases only for entries that are recorded as changed since last update, these entries could not be identified as of fungal origin. ACNUC people, when informed answered that they would correct this problem in the near future.

\section{Conclusion}

Analyses of responses found by SRS, Entrez, ACNUC and a dedicated script showed:

1/Entrez capabilities for retrieving the proper data set are limited as specific FT lines cannot be assigned. Also, subsequences are not automatically extracted. Both limitations render Entrez unfit for the purpose sought.

2/SRS retrieved some entries not present in EmblEx, all were false positives that mostly consisted of sub-entries annotated as "internal transcribed spacer region 1". Although in this case the answer is right, this is not a proper answer and could lead in other cases to unwanted sequences. More important, the day of the final demonstration query, SRS at Infobiogen returned a wrong dataset, with no error message, due to indexing problems. EmblEx retrieved some entries that SRS missed, mostly due to a problem with the presence of white spaces and parentheses, as in accession numbers AB176462 \& AB176463, with a misc_rna feature containing "contains internal transcribed spacer 1,2, 5.8S ribosomal RNA". SRS could be used to index phrases, but the administrators are (usually) not doing it, one reason probably is that using the exact phrase is only appropriate when one already knows that phrase. Most of the time users have much fuzzier queries - at least until they have found what they want and can start to refine it. Finally, SRS showed problems when the amount of memory necessary to run the queries turned out to be too big. On the EBI server for
Table 4: Annotations of the 64 responses of SRS not found by EmbIEx.

53 internal transcribed spacer region I

7 Internal transcribed spacer (ITS) I region

I transcribed spacer I, 5.8S ribosomal RNA, internal...

2 RNA, transcribed spacer I, 5.8S ribosomal RNA, internal...

I contains nternal transcribed spacer I, 5.8S ribosomal RNA, internal...

example, the Q1 query indicated above but run on Embl (Emblrelease + Emblnew) returned a memory allocation error message, which is presumably not due to SRS per se but to the available memory of the hardware used to run SRS.

3/ACNUC had no problem of memory of any sort and was fast as long as scanning the FT lines was not required. It retrieved entries not found by the Perl script; they corresponded to keywords for other features (such as "snorna" and "precursor_rna"), since we did not use a search in specific features for reasons mentionned above.

4/If the data obtained are of importance, it is safer to query different servers and compare the results obtained.

5/Extensive analyses of results provided by the public tools can detect subtle bugs or problems due to the semistructured form of the EMBL/GenBank entries.

In a recent work, D'Addabbo and coworkers [26] also noticed a problem in extracting data from feature definition with SRS; their solution (GeneRecords) was to build extraction procedures to import GenBank entries in a FileMaker $^{\circ}$ database. Compared to GeneRecords, EmblEx makes use of non proprietary software and runs under every OS (developed under Linux and MS-Windows for compatibility). It also renders it possible to import directly in the BioSQL format.

A script is obviously not as fast as ACNUC or SRS, since no index tables are used, but is more flexible and precise. It is however possible to first use SRS or ACNUC to extract a large number of entries and then run EmblEx on these entries for a final finely tuned analysis, combining speed of extraction with precision. In particular EmblEx allows users to specify a search order in the annotations, making sure for example that if both "ITS1" and "ITS2" are in the "source" field, and "ITS1" in the "misc_rna" field, only the latter sequence will be properly extracted (which could be done using ACNUC and a dedicated script).

In conclusion, the advantages of EmblEx are in particular i) easy modifications of the code for a particular use, ii) use of the BioSQL standard, therefore allowing subsequent use of public routines (BioJava, BioPython and 
BioPerl), iii) very precise queries through a graphical interface or the command line and, iv) simple local use or access to a distant server through the web.

More details can be found in the html documentation provided with EmblEx.

\section{Availability and requirements}

- Project name: EmblEx

- Project home page: http://bioinfo.unice.fr/ softwares en.htm

- Operating system(s): Windows, Unix

- Programming language: Perl, HTML

- Other requirements: Apache 1.x or higher, MySQL 3.x or higher

- License: Open Source http://ftp.bioinformatics.org/ pub/emblex/emblex.html

\section{Authors' contributions}

ML and OC implemented the software (Perl scripts) and the graphical user interface, and were the primary authors of the manuscript. RC participated in design and coordination and wrote the Python scripts. All authors read and approved the final manuscript.

\section{Acknowledgements}

This work was in part funded by the"ACI Microbiologie fondamentale et appliquée" (2003). We thank R. Arkowitz for careful reading, and two reviewers for suggestions that helped improving this manuscript.

\section{References}

I. EBI statistics [http://www3.ebi.ac.uk/Services/DBStats/]

2. Altschul SF, Gish W, Miller W, Myers EW, Lipman DJ: Basic local alignment search tool. J Mol Biol 1990, 215:403-410.

3. Slater GS, Birney E: Automated generation of heuristics for biological sequence comparison. BMC Bioinformatics 2005, 6:31.

4. Gouy M, Gautier C, Attimonelli M, Lanave C, di Paola G: ACNUC a portable retrieval system for nucleic acid sequence databases: logical and physical designs and usage. Comput Appl Biosci 1985, I: 167-172

5. Schuler GD, Epstein JA, Ohkawa H, Kans JA: Entrez: molecular biology database and retrieval system. Methods Enzymol 1996, 266:141-162.

6. Etzold T, Argos P: SRS - an indexing and retrieval tool for flat file data libraries. Comput Appl Biosci 1993, 9:49-57.

7. Location of EMBL release files (EBI) [ftp://ftp.ebi.ac.uk/pub/ databases/embl/release/]

8. BioSQL [http://cvs.bioperl.org/cgi-bin/viewcvs/viewcvs.cgi/biosqlschema/sql/?crsroot=biosql]

9. bioperl-db script [http://bio.perl.org/Core/Latest/index.shtml]

10. Location to download Emblex [http://bioinfo.unice.fr/ softwares en.htm]

II. Sudagidan M, Yenidunya AF, Gunes H: Identification of staphylococci by $16 \mathrm{~S}$ internal transcribed spacer rRNA gene restriction fragment length polymorphism. J Med Microbiol 2005, 54:823-826.

12. Park H, Jang H, Song E, Chang CL, Lee M, Jeong S, Park J, Kang B, Kim $C$ : Detection and genotyping of Mycobacterium species from clinical isolates and specimens by oligonucleotide array. J Clin Microbiol 2005, 43: I 782-1788.

13. Conrads G, Citron DM, Tyrrell KL, Horz HP, Goldstein EJ: I6S-23S rRNA gene internal transcribed spacer sequences for analysis of the phylogenetic relationships among species of the genus Porphyromonas. Int J Syst Evol Microbiol 2005, 55:607-6I3.

14. Hirschhauser S, Frohlich J, Gneipel A, Schonig I, Konig H: Fast protocols for the 5S rDNA and ITS-2 based identification of Oenococcus oeni. FEMS Microbiol Lett 2005, 244: I65-I7I.

15. Suzuki MT, Preston CM, Beja O, de la Torre JR, Steward GF, DeLong EF: Phylogenetic screening of ribosomal RNA gene-containing clones in Bacterial Artificial Chromosome (BAC) libraries from different depths in Monterey Bay. Microb Ecol 2004, 48:473-488.

16. Nubel U, Schmidt PM, Reiss E, Bier F, Beyer W, Naumann D: Oligonucleotide microarray for identification of Bacillus anthracis based on intergenic transcribed spacers in ribosomal DNA. FEMS Microbiol Lett 2004, 240:21 5-223.

17. Medina A, Mateo R, Lopez-Ocana L, Valle-Algarra FM, Jimenez M: Study of Spanish grape mycobiota and ochratoxin A production by Isolates of Aspergillus tubingensis and other members of Aspergillus section Nigri. Appl Environ Microbiol 2005, 7l:4696-4702.

18. Hsiao CR, Huang L, Bouchara JP, Barton R, Li HC, Chang TC: Identification of medically important molds by an oligonucleotide array. I Clin Microbiol 2005, 43:3760-3768.

19. Bjelland T, Ekman S: Fungal diversity in rock beneath a crustose lichen as revealed by molecular markers. Microb Ecol 2005 , 49:598-603.

20. Rakeman JL, Bui U, Lafe K, Chen YC, Honeycutt RJ, Cookson BT: Multilocus DNA sequence comparisons rapidly identify pathogenic molds. J Clin Microbiol 2005, 43:3324-3333.

21. Nagao K, Ota T, Tanikawa A, Takae Y, Mori T, Udagawa S, Nishikawa $\mathrm{T}$ : Genetic identification and detection of human pathogenic Rhizopus species, a major mucormycosis agent, by multiplex PCR based on internal transcribed spacer region of rRNA gene. J Dermatol Sci 2005, 39:23-31.

22. Hinrikson HP, Hurst SF, Lott TJ, Warnock DW, Morrison CJ: Assessment of ribosomal large-subunit DI-D2, internal transcribed spacer $I$, and internal transcribed spacer 2 regions as targets for molecular identification of medically important Aspergillus species. I Clin Microbiol 2005, 43:2092-21 03.

23. Kumar M, Shukla PK: Use of PCR targeting of internal transcribed spacer regions and single-stranded conformation polymorphism analysis of sequence variation in different regions of rrna genes in fungi for rapid diagnosis of mycotic keratitis. J Clin Microbiol 2005, 43:662-668.

24. List of servers offering SRS [http://downloads.lionbio.co.uk/pub licsrs.html]

25. ACNUC client (on the pbil server) [http://pbil.univ-lyonl.fr/ software/query.html]

26. D'Addabbo P, Lenzi L, Facchin F, Casadei R, Canaider S, Vitale L, Frabetti F, Carinci P, Zannotti M, Strippoli P: GeneRecords: a relational database for GenBank flat file parsing and data manipulation in personal computers. Bioinformatics 2004, 20:2883-2885.

Publish with Bio Med Central and every scientist can read your work free of charge

"BioMed Central will be the most significant development for disseminating the results of biomedical research in our lifetime. "

Sir Paul Nurse, Cancer Research UK

Your research papers will be:

- available free of charge to the entire biomedical community

- peer reviewed and published immediately upon acceptance

- cited in PubMed and archived on PubMed Central

- yours - you keep the copyright 